\section{Inquéritos Nacionais de Saúde: experiência acumulada e proposta para o inquérito de saúde brasileiro}

\section{Deborah Carvalho Malta' \\ Maria do Carmo Leal² \\ Maria Fernanda Lima Costa ${ }^{3}$ \\ Otaliba Libânio de Morais Neto ${ }^{4}$}

1 Coordenação Geral de Doenças e Agravos Não Transmissíveis-Departamento de Análise de Situação da Saúde, Secretaria de Vigilância em Saúde, Ministério da Saúde. Escola de Enfermagem-UFMG

${ }^{2}$ Fundação Oswaldo Cruz, Ministério da Saúde.

${ }^{3}$ Núcleo de Estudos em Saúde Pública e Envelhecimento da Fundação Oswaldo Cruz e Universidade Federal de Minas Gerais. Centro Colaborador da Secretaria de Vigilância à Saúde em Saúde do Idoso e Epidemiologia do Envelhecimento.

${ }^{4}$ Departamento de Análise de Situação da Saúde, Secretaria de Vigilância em Saúde, Ministério da Saúde. Instituto de Patologia Tropical e Saúde Pública-UFG.

Correspondência: Deborah Carvalho Malta - Secretaria de Vigilância em Saúde - Ministério da Saúde 55 - 61 - 33153784 - Esplanada Dos Ministérios - Ed-Sede - Sala 142 - CEP 70058-900 Brasília DF. E-mail: deborah.malta@saude.gov.br

\section{Resumo}

As informações oriundas das fontes de dados secundários dos sistemas de informação em saúde existentes são fundamentais, mas insuficientes para responder às necessidades atuais da gestão do Sistema Único de Saúde na identificação dos problemas e necessidades de saúde da população brasileira. Os inquéritos populacionais são instrumentos utilizados como subsídios à formulação e avaliação das políticas públicas, tornando-se crescente a sua utilização nos diversos países como ferramenta de apoio ao planejamento em saúde. O artigo realiza uma breve revisão das iniciativas de realização de inquéritos no âmbito internacional e no Brasil, e propõe um roteiro para a realização do Inquérito Nacional de Saúde.

Palavras Chaves: Inquérito de Saúde, Sistema Único de Saúde; Informação em Saúde 
Abstract

The information from secondary data sources of existing health information systems are fundamental but insufficient to answer to the current needs of management in the Brazilian Health System (SUS) to identify problems and health needs of the population of Brazil. The population surveys are instruments used as subsidies for formulating and evaluating public policy, its use is increasing in several countries as a support tool in planning health. The paper makes a short revision of survey initiatives in International scope and in Brazil, it proposes a schedule for the realization of the National Health Survey.

Keywords: Health Survey, Brazilian Health System (SUS), Health Information

\section{Introdução}

As informações em saúde são cada vez mais essenciais para o planejamento, a programação, o monitoramento e a gestão das intervenções em saúde coletiva e individual, principalmente quando se considera o contexto de mudanças do padrão epidemiológico, ampliação do conceito saúde-doença, bem como a incorporação das atividades de promoção da saúde. As informações oriundas das fontes de dados secundários dos sistemas de informação em saúde são fundamentais, mas insuficientes para responder às necessidades da gestão. Daí a importância crescente de inquéritos populacionais, entendidos como componentes fundamentais para um sistema nacional de informações em saúde.

Inquéritos Nacionais de Saúde (INS) permitem conhecer o perfil de saúde e a distribuição dos fatores de risco em uma população, com atualização periódica e comparações seqüenciadas no tempo e entre áreas geográficas. O primeiro Inquérito Nacional de Saúde pode se constituir numa linha de base para avaliações subseqüentes.

Uma dimensão importante dos inquéritos refere-se à possibilidade de correlacionar os agravos e problemas de saúde com as condições sócio-ambientais e mesmo com a percepção do estado de saúde pelos indivíduos, permitindo a descrição e quantificação das iniqüidades em saúde, tanto relacionadas à ocorrência de doenças quanto à exposição a riscos. Problemas de saúde menos graves ou pouco sintomáticos, bem como a identificação de grupos com dificuldade de acesso aos serviços de saúde podem ser também identificados por meio de inquéritos de saúde.

Além disto, os INS podem se constituir em instrumentos de avaliação das políticas públicas implantadas, o grau de sucesso alcançado por elas, bem como identificar grupos sociais e geográficos excluídos ou pouco atingidos. Da mesma forma, se devidamente planejado, os INS podem permitir a comparação do desempenho 
entre Sistemas de Saúde com organizações sociais e institucionais distintas, como, por exemplo, tem sido publicado sobre os sistemas de saúde norte americano e canadense ${ }^{1}$.

Não menos importante nas sociedades contemporâneas é a necessidade de se conhecer a carga de doença oriunda de hábitos não saudáveis e o cálculo dos custos para o sistema de saúde e a sociedade, subsidiando políticas de intervenção legal sobre problemas, como consumo de alimentos, cigarros e de álcool (viabilizado pela mescla de informações oriundas dos inquéritos sobre exposição, absenteísmo ao trabalho decorrente, por exemplo, de alcoolismo (casos leves), com as informações oriundas dos Sistemas de Informação em Saúde como duração das internações e procedimentos, bem como os custos decorrentes da perda de vidas (óbitos).

Um dos fatores limitantes no uso rotineiro dos inquéritos consiste nos custos elevados, além dos processos de desenho das amostras e dos procedimentos de análise serem metodologicamente complexos, exigindo-se tempo e capacidade técnica para planejamento e execução.

O presente artigo propõe-se a realizar uma breve revisão das iniciativas de realização de inquéritos no âmbito internacional e no Brasil, bem como propor um roteiro para a realização do Inquérito Nacional de Saúde.

\section{Inquéritos no âmbito Internacional}

Nos países de alta renda, desde a década de 1960, os inquéritos populacionais são instrumentos utilizados para a formulação e avaliação das políticas públicas. Existem vários exemplos internacionais de inquéritos de fatores de risco, morbidade, medidas bioquímicas dentre outros. $\mathrm{Na}$ Inglaterra, além dos censos demográficos decenais que incluem informações sobre saúde, existem inquéritos contínuos, sendo os mais importantes, o General Health Survey (GHS), que vai a campo desde 1971, e o Health Survey for England (HSE), im- plantado em 19932. Nos Estados Unidos, o National Health Interview Survey (NHIS) que coleta informações auto-referidas sobre a ocorrência anual de doenças agudas e acidentes, existência de doenças crônicas e incapacidades, e utilização de serviços de saúde. O National Health and Nutrition Examination Survey (NHANES) coleta dados como exame físico, medidas bioquímicas e fisiológicas. No Canadá, o National Population Health Survey (NPHS), tem dados sobre condições de saúde, uso de serviços de saúde, condições de trabalho e hábitos de vida que influenciam as condições de saúde. Entretanto, mesmo existindo múltiplos inquéritos nos países desenvolvidos ainda existem problemas como a articulação dos mesmos (superposição e duplicação de dados, falta de padronização de terminologias e definições das variáveis), comprometendo a comparação de dados de diferentes bases de dados ${ }^{2}$.

Nos países de baixa e média renda, a prática de inquéritos é recente, tendo caráter mais episódico e são baseados em modelos desenvolvidos por organismos internacionais como a Organização Mundial da Saúde (OMS), Organização Panamericana da Saúde (OPAS), dentre outros. A OMS tem formulado e aplicado o World Health Survey (WHS), visando superar a falta de dados existentes principalmente nos países em desenvolvimento ${ }^{3}$.

Nas últimas décadas, a OMS e OPAS tem incentivado o uso de inquéritos de fatores de risco e protetores das Doenças Crônicas Não Transmissíveis nos países, o que tem ampliado sua utilização ${ }^{4}$. Este tipo de informação torna-se útil para o monitoramento das ações implementadas.

Ênfase especial tem sido dada para os inquéritos em grupos específicos que tem sido alvo de monitoramento mais freqüente, seja pela possibilidade de detecção precoce de agravos e oportunidade de estabelecer políticas de promoção à saúde $\mathrm{e}$ prevenção, como, por exemplo, entre jovens, ou, pela maior morbi-mortalidade entre idosos, ou grupos de pessoas que 
apresentam patologias que atuam como fatores de risco para outras doenças crônicas não transmissíveis, como diabéticos e hipertensos.

A OMS tem incentivado inquéritos entre a população jovem em diversas regiões do mundo, por ser custo-efetivo a prevenção de doenças crônicas nesta faixa etária. Os adolescentes estão expostos a novas práticas e comportamentos, exposição a diversas situações e riscos como o tabagismo, consumo de álcool, alimentação inadequada e sedentarismo. Assim o monitoramento destes fatores poderá subsidiar políticas públicas. A OMS conjuntamente com o Centro para Controle e Prevenção de Doenças (CDC), desenvolveu uma metodologia para esta finalidade, o Global School-based Student Health Survey (GSHS) ${ }^{5}$. Esta estratégia investiga fatores de risco comportamentais e de proteção em adolescentes de 13 a 15 anos, tem sido implantada ou está em processo de implantação em 22 países. O Youth Health Risk Beahaviour Surveillance System (YRBSS) é realizado nos Estados Unidos a cada dois anos desde 1990 pelo CDC, estuda escolares de 12 a 21 anos de idade. Monitora os principais fatores de risco relacionados às causas de morte, incapacidades e problemas sociais entre os jovens americanos: tabagismo, alimentação, consumo de álcool e outras drogas, atividade física, comportamento sexual de risco e comportamentos associados com acidentes e violências ${ }^{6}$. O Health Beahaviour in School-aged Children Study (HBSC) é um sistema de monitoramento iniciado na Finlândia, Noruega e Inglaterra em 1982, e atualmente é realizado outros países da Europa em cooperação com a OMS. Estuda escolares de 11 a 15 anos de idade e também investiga comportamentos de risco relacionados com a saúde ${ }^{7}$.

Outro segmento que tem recebido crescente atenção é a população idosa. Isso se justifica porque é a população que mais cresce no mundo, a que apresenta maior carga de doenças e de incapacidades e a que mais usa serviços de saúde. Embora não exista um modelo de inquérito de saúde para essa população com abrangência mundial, existem iniciativas importantes, sobretudo, nas Américas e na Europa. Nos Estados Unidos, por exemplo, o Centers for Disease Control and Prevention ${ }^{8}$ utiliza dados de inquéritos de saúde para manter um sistema nacional de informações sobre as condições de saúde da população idosa. Esse sistema é alimentado por informações obtidas por meio de inquéritos de saúde, tais como o National Health Interview Survey (NIHS), o National Health and Nutrition Examination Survey (NHANES), o Nursing Home Residents, o Medicare Current Beneficiaty Survey, o Behavioral Risk Factor Surveillance (BRFSS) e o Longitudinal Study of Aging $(L O A S)^{8}$. No Canadá, o Health Canada utiliza informações obtidas por meio de inquéritos de saúde para monitorar as condições de saúde da população idosa ${ }^{9}$. Esses sistemas constituem bons exemplos de como os inquéritos nacionais de saúde podem ser úteis para subsidiar políticas públicas para a população idosa. Mais recentemente, sob os auspícios da Organização Pan Americana de Saúde, foi realizado um inquérito multicêntrico para examinar as condições de saúde dos idosos na América Latina e no Caribe ${ }^{10}$. No Brasil, estudos utilizando dados dos suplementos de saúde da Pesquisa Nacional por Amostras de Domicílio (PNAD 1998 e 2003), foram realizados para examinar as condições de saúde da população idosa brasileira ${ }^{11}$, para determinar as tendências dessas condições $^{12}$ e para examinar a influência da situação da situação sócio econômica sobre as condições de saúde dos idosos ${ }^{13,14}$.

\section{A experiência brasileira de informação em saúde e de inquéritos}

No Brasil, as informações em saúde são obtidas principalmente pelos Sistemas Nacionais de Informação em Saúde gerenciados pelo Sistema Único de Saúde e aquelas obtidas pelos inquéritos regio- 
nais ou Nacionais realizados no Brasil principalmente a partir da década de 80 .

O SUS dispõe de sistemas de informação sobre mortalidade (SIM), doenças de notificação compulsória e outros agravos (SINAN), produção de serviços e atendimentos ambulatoriais (SIA), internações hospitalares (SIH), nascidos vivos (SINASC), informação da atenção básica (SIAB), dentre outros.

Com relação aos inquéritos, o IBGE (Instituto Brasileiro de Geografia e Estatística) é o responsável pela maioria das pesquisas domiciliares em curso no Brasil. O IBGE, criado em 1938, realiza os censos nacionais e inquéritos, como a PNAD (Pesquisa Nacional de Amostra de Domicílios), que produzem dados demográficos, estatísticas vitais, levantamentos sócio-econômicos. Os dados censitários, coletados decenalmente, fornecem importantes informações demográficas, ambientais, sócio econômicas, emprego, renda, trabalho, fecundidade, mortalidade e características dos domicílios. No período entre os censos são realizadas diferentes coletas de dados sobre diversos temas como o levantamento da rede de assistência a saúde, dados sobre orçamento familiar, saúde e nutrição, saúde reprodutiva, práticas de contracepção, migração, trabalho, educação, trabalho infantil, dentre outros.

A Pesquisa Nacional por Amostras de Domicílio (PNAD/IBGE), é um inquérito de base populacional, realizado anualmente, com abrangência nacional e produz informações sobre características demográficas, habitação, educação, trabalho e rendimentos da população brasileira. Em 1998 e 2003, a pesquisa incluiu um suplemento sobre saúde, estando prevista a sua repetição em 2008. O suplemento de saúde da PNAD é, possivelmente, o inquérito de saúde mais abrangente já realizado na população brasileira com 14 ou mais anos de idade. Esse inquérito apresenta diversas vantagens. As principais delas são o grande tamanho da amostra - permitindo, por exemplo, análises separadas para homens e mulheres, assim como para idosos e adultos mais jovens, entre outras, a abrangência nacional e a sua periodicidade que possibilita o acompanhamento das tendências dos indicadores. Além disso, por ser parte de uma pesquisa mais ampla sobre condições sócio econômicas, ela possibilita uma infinidade de estudos sobre fatores associados às condições de saúde e uso de serviços de saúde, como já foi demonstrado por meio de várias publicações acadêmicas sobre o tema. Ela também produz informações que podem ser utilizadas para monitoramento das condições de saúde, e aspectos a ela relacionada, da população brasileira. Por outro lado, ela apresenta limitações, por exemplo, a unidade amostral é o domicílio e não o indivíduo, ou seja, todos os moradores com 14 ou mais anos de idade são selecionados. Isso faz com que o uso de respondentes substitutos nessa pesquisa seja alto (30\%) e não se sabe como isso influencia os resultados observados. Uma outra limitação é que, por ser o suplemento de uma pesquisa maior, o tamanho do questionário é limitado. De qualquer forma, os dados produzidos pela PNAD constituem importante fonte de informação para o planejamento de políticas sociais.

Outra pesquisa realizada pelo IBGE é a Pesquisa sobre Orçamento Familiar (POF), iniciada no final da década de 80 e que levanta os gastos das famílias brasileiras com relação a vários itens incluindo gastos com saúde. Em sua última versão incorporou medidas antropométricas ${ }^{15}$.

Além dos inquéritos produzidos pelo IBGE, apresentaram relevância as seguintes pesquisas: a Pesquisa Nacional sobre Saúde e Nutrição (PNSN) de 1989, a Demographic and Health Survey (DHS) realizada pela Benfam em 1986, 1991 e 1996 e a Pesquisa sobre Padrão de Vida (PPV) realizada pelo Banco Mundial nas regiões Nordeste e Sudeste ${ }^{15}$.

Como parte de um projeto desenvolvido pela Organização Mundial da Saúde (OMS) dirigido à avaliação de desempenho dos sistemas de saúde dos países membros, a Pesquisa Mundial de Saúde - inqué- 
rito em 5000 indivíduos com 18 anos e mais de idade - foi realizada no Brasil, no ano de 2003. Utilizando-se instrumento modular, foram abordados os seguintes aspectos de saúde: condições socioeconômicas; descrição do estado de saúde (auto-avaliação da saúde e limitações de atividades); fatores de risco (fumo, álcool, atividade física, nutrição, fatores ambientais); situações crônicas (diagnóstico e tratamento); situações agudas (causas externas); assistência pré-natal; saúde infantil; e avaliação da assistência recebida (ambulatorial ou hospitalar) de acordo com as expectativas do usuário. A oportunidade de execução da pesquisa no Brasil significou a oportunidade de suprir informações para a construção de indicadores para avaliar o desempenho do sistema nacional de saúde, além de possibilitar o estabelecimento de parâmetros consistentes para avaliar as desigualdades socioeconômicas da situação de saúde da população brasileira $^{16}$.

O Ministério da Saúde tem apoiado inquéritos desenvolvidos pelo IBGE, como por exemplo, a PNAD (suplemento saúde), POF e AMS (Assistência Médica Sanitária), além de apoiar e desenvolver diversos inquéritos com temas específicos. Destaca-se o papel da Secretaria de Vigilância em Saúde (SVS), e o antigo CENEPI (Centro Nacional de Epidemiologia) na indução dos inquéritos nacionais em saúde. Documento produzido pelo CENEPI em 2000 destacava o papel do órgão como sendo responsável por "Promover uma discussão nacional sobre inquéritos nacionais para pactuar temas, metodologias, regularidade, abrangência, padronização da organização e gestão de bancos de dados e estratégias de análise" ${ }^{17}$.

Nestes últimos anos estão sendo desenvolvidos inquéritos importantes no campo das doenças transmissíveis, tais como: o inquérito sorológico hepatites, já concluído nas regiões Nordeste e Centrooeste, o inquérito sorológico doença de chagas em fase de conclusão e o inquérito cobertura vacinal em fase de liberação de recursos financeiros.
Com relação às doenças e agravos não transmissíveis (DANT) e visando a estruturação de um sistema de vigilância, tem sido desenvolvido um conjunto de ações que possibilitam conhecer a distribuição, magnitude e tendência dessas doenças, por meio de fontes secundárias de informações e no monitoramento contínuo dos fatores de risco, identificando seus condicionantes sociais, econômicos e ambientais, com o objetivo de subsidiar o planejamento, execução e avaliação da prevenção e controle das mesmas. O monitoramento das DANT pressupõe o fluxo sistemático de dados secundários e primários. As principais fontes de dados são os sistemas de informação em mortalidade e internações hospitalares e os inquéritos de saúde periódicos e especiais. Visando disponibilizar o país de informações sobre estes agravos e os seus fatores de risco $(\mathrm{FR})^{18}$.

Com esse objetivo foram realizados os seguintes inquéritos de abrangência nacional: o Inquérito Domiciliar sobre Comportamentos de Risco e Morbidade Referida de Doenças e Agravos Não-transmissíveis $(2002-2005)^{19}$ realizado por meio de parceria entre a SVS e o Instituto Nacional do Câncer (INCA) para 18 capitais brasileiras; o Inquérito Nacional sobre Fatores de Risco e Proteção de Doenças não Transmissíveis por meio de entrevistas telefônicas (VIGITEL) realizado em $2006^{20}$, com característica de um levantamento contínuo anual); a Pesquisa Nacional de Saúde do Escolar (PENSE), que encontra-se em fase de planejamento do campo e apresenta como objetivo o monitoramento dos fatores de risco e proteção na população jovem das capitais brasileiras; e o Sistema de Vigilância de Violências e Acidentes (VIVA) implantado em 39 municípios brasileiros em 2006, com o objetivo de identificar o perfil das violências nos hospitais de urgências localizados nesses municípios ${ }^{18,21}$.

Outras iniciativas em curso consistem na inclusão pelo IBGE, no suplemento saúde da PNAD/2008, de um módulo específico sobre fatores de risco para doenças e agravos não transmissíveis contendo ques- 
tões referentes a pratica de atividade física; tabagismo, acidentes e violências, bem como acréscimo de questões referentes à acesso dos serviços do SUS no módulo de detecção precoce de câncer do colo do útero e mama. Além disso, a inserção na POF de 2008 da pesquisa sobre consumo alimentar e de medicamentos.

Merecem ainda destaque duas iniciativas ocorridas no ano de 2006: a criação do Comitê Técnico Interinstitucional no âmbito da Rede Interagencial para informações em Saúde (RIPSA) com o objetivo de discutir o modelo de inquéritos em Saúde para o Brasil adequado às demandas do país e a criação da Comissão Intraministerial de Inquéritos, por meio da Portaria Ministerial No 3.175 , composta por várias Secretarias do Ministério da Saúde (MS) e coordenada pela Secretaria de Ciência e Tecnologia. Esta comissão visa articular a demanda do Ministério da Saúde junto ao IBGE e o planejamento dos conteúdos para a PNAD e $\mathrm{POF}^{22}$.

\section{Planejamento do Inquérito Nacional de Saúde}

Para a construção da proposta do Inquérito Nacional de Saúde é importante considerar duas dimensões: aspectos relacionados à gestão e decisão política e planejamento e execução do Inquérito Nacional de Saúde (INS).

No que se refere à gestão, há que envolver todas as áreas do MS, organizando a definição dos temas que o Inquérito $\mathrm{Na}$ cional de Saúde deve abordar. O estudo deve ser capaz de responder às lacunas das informações existentes no âmbito do SUS, de forma a identificar e fornecer informações sobre os determinantes e condicionantes de saúde, as necessidades de saúde, os usos dos serviços de saúde e sobre as desigualdades existentes na população brasileira nos itens citados anteriormente. Outras decisões importantes para o Inquérito Nacional em Saúde consistem na definição do montante de recursos financeiros que podem ser alocados para a sua realização, a sua abrangência geográfica (macro-região, unidade federada, ou região metropolitana), e, como desdobramento, qual o tamanho da amostra e a definição das parcerias para sua realização.

Torna-se importante envolver a ABRASCO, Instituições Acadêmicas, IBGE, CONASS e CONASEMS como parceiros neste processo. $\mathrm{O}$ apoio das instituições de ensino e pesquisa é essencial no planejamento, desenho metodológico, execução e análise dos dados, dentre outros.

Com relação à responsabilidade pela condução da pesquisa, é importante considerar a grande experiência e capacidade técnica e operacional do IBGE na coordenação dos inquéritos no Brasil, garantindo a continuidade das várias pesquisas sob sua coordenação. No momento, o IBGE está em processo de implantação do sistema integrado de pesquisas domiciliares, introduzindo a amostra mestra, que criará condições de atender a novas demandas, a continuidade e integração das pesquisas. Estas mudanças abrem a possibilidade de maior integração com as demandas da saúde, temas, amostragem, garantia de continuidade dos inquéritos, sua sustentabilidade e comparabilidade temporal ${ }^{24}$.

Como decisões políticas e de gestão fundamentais, ressalta-se a responsabilidade pela coordenação executiva do INS, a sua inserção nos Planos Plurianual e Nacional de Saúde de forma a garantir a sua viabilização. Além disso, a definição da periodicidade do levantamento, tendo em vista que o Inquérito Nacional deve ser o carro chefe da organização de um Sistema Nacional de Inquéritos Populacionais no âmbito do SUS.

Do ponto de vista do Planejamento e Execução, torna-se necessário avançar na definição da metodologia, amostragem adequada (desenho da amostra e número de domicílios/pessoas), se serão realizadas sub-amostras para possibilitar a obtenção de medidas físicas e coleta de material biológico para diagnóstico de morbidades específicas. A abordagem dos entrevista- 
dos quando da assinatura do texto do consentimento esclarecido deve incluir a autorização ampla para o uso de material biológico para exames futuros, não previstos, tomando como referência a experiência dos EUA, que regulamentou estas questões no Comitê Nacional de Bioética para pesquisa com seres humanos em $1999^{24}$.

Neste último ponto cabe a discussão da proposta de escopo de inquéritos proposta pela $\mathrm{OMS}^{4}$, na qual se trabalha com passos seqüenciais, abordando no primeiro step (passo), as entrevistas, e seguidas nos passos seguintes pela inserção de medidas (pressão arterial, antropometria e exames de laboratório). Há que se avançar na forma de coleta e instrumentos de coleta; bem como na logística do trabalho de campo e deslocamentos das equipes e materiais biológicos, por fim no planejamento da proposta de processamento e análise dos dados coletados.

Por fim é necessária a definição de um grupo executivo de trabalho, que desenvolva uma proposta para submeter ao Ministério da Saúde para deliberação sobre a realização do Inquérito Nacional de Saúde.

\section{Conclusão}

Políticas públicas em saúde devem se apoiar em informações objetivas respaldadas por evidências científicas. A Saúde Pública e a Epidemiologia têm um importante papel nesse processo, seja por meio do desenvolvimento de pesquisas, como pela coleta sistemática de informações oriundas dos sistemas de vigilância que possibilita a avaliação sistemática de dados sobre magnitude, escopo, características e conseqüências das doenças. Os inquéritos em saúde são importantes tam- bém para produzir informações que sensibilizem os gestores públicos responsáveis pela implementação das políticas públicas no que se refere aos principais problemas e as iniqüidades vividas pela população. A tradução de informações científicas sobre evidências para políticas e práticas que protejam os usuários é fundamental nesse processo ${ }^{18}$.

A realização do Inquérito Nacional em Saúde será um instrumento para suprir lacunas no conhecimento da realidade brasileira no campo da saúde pública, pois possibilitará: (i) conhecer aspectos estratégicos da saúde pública brasileira, não contemplados na PNAD, como, por exemplo, o acesso a políticas promotoras da saúde; (ii) gerar hipóteses sobre mecanismos geradores de doença, inclusive aqueles decorrentes nas iniqüidades sociais em saúde; (iii) obter informações de base populacional sobre medidas físicas (antropometria, pressão arterial, e outras a serem definidas); (iv) ter uma linha de base para realização de estudos longitudinais, que utilizam os sistemas de informações existentes como o SIM e SIH para seguimento em sub-amostra de populações previamente definidas; (v) avaliar a satisfação do cliente, o usuário do SUS quanto a atenção recebida no sistema; (vi) criar um rico espaço para o desenvolvimento de métodos, tais como a aceitabilidade, validade e reprodutibilidade de instrumentos sumarizados, avaliação da importância dos não respondentes de entrevistas entre os amostrados na modificação dos resultados do estudo e custos para encontrá-los. Enfim, será uma ferramenta de apoio ao planejamento em saúde e para a formulação de políticas públicas sociais integradas e articuladas.

\section{Referências Bibliográficas}

1. Steinberg, KK. Ethical challenges at the beginning of the millennium. Stat. Med, 2001; vol. 20, no. 9-10: 1415-9.

2. Viacava, F. Informações em saúde: a importância dos inquéritos populacionais. Ciênc. saúde coletiva, 2002; vol.7, no. 4: 607-621.
3. World Health Organization. World Health Survey. 2003. http://www.who.int/healthinfo/survey/en/ acessado em fevereiro de 2007.

4. World Health Organization. Preventing Chronic Diseases a Vital Investment. Switzerland. 2005. www.who.int/chp/ chronic_disease_report/en/ 
5. World Health Organization. Global school-based student health survey. 2005. Disponível em: http://www.who.int/ school_youth_health/assessment/gshs/implementation/ en/Acessado em: 2005. Maio

6. Centro de Controle e Prevenção de Doenças. Youth Risk Behavior Surveillanc. United States, 2005 . Disponível em: http://www.cdc.gov/HealthyYouth/yrbs/ about_yrbss.htm. Acessado em 2005. Jan.

7. Health Beahaviour in School-aged Children Study (HBSC). World Health Organization Collaborative Cross National Study. WHO Regional Office for Europe. http:// www.hbsc.org/ http://www.hbsc.org/overview.html. Acessado em março de 2007.

8. Centers for Disease Control and Prevention. National Center for Health Statistics. Trends in Health and Aging. 2007. Disponível em: www.cdc.gov/nchs/agingact.htm. Acessado em: Jun 2007.

9. Canadá. Government of Canadá. National Advisory Council on Aging. Report Card Seniors in Canada, 2001. $50 \mathrm{p}$.

10. Palloni A, McEniry M. Aging and Health Status of Elderly in Latin America and the Caribbean: Preliminary Findings. J Cross Cult Gerontol. 2006 Oct 5 [Epub ahead of print]

11. Lima-Costa MF, Barreto SM e Giatti L. Condições de saúde, capacidade funcional, uso de serviços de saúde e gastos com medicamentos da população idosa brasileira: um estudo descritivo baseado na Pesquisa Nacional por Amostras de Domicílio (PNAD 98) Cad Saúde Pública 2003; 19: 735-43,

12. Lima-Costa MF, Loyola Filho AI, Matos DL. Tendências nas condições de saúde e uso de serviços de saúde entre idosos brasileiros: um estudo baseado na Pesquisa Nacional por Amostras de Domicílio $(1998,2003)$. Cad Saúde Pública (em publicação).

13. Lima-Costa MF, Barreto SM \& Giatti L. A situação sócio econômica afeta igualmente a saúde de idosos e adultos mais jovens no Brasil? Um estudo utilizando dados da Pesquisa Nacional por Amostras de Domicílio (PNAD 98). Ciênc. Saúde Coletiva 2002; 7: 285-296.

14. Lima-Costa MF, Matos DL, Camarano AA. Evolução das desigualdades sociais em saúde entre idosos e adultos brasileiros: um estudo baseado na Pesquisa Nacional por Amostras de Domicílio (PNAD) 1998, 2003. Ciênc. Saúde Coletiva 2006; 11: 941-50.
15. Viacava F, Dachs N, Travassos C. Os inquéritos domiciliares e o Sistema Nacional de Informações em Saúde. Ciênc. Saúde Coletiva 2006; 11, no. 4: 863-69.

16. Szwarcwald CL, Viacava F. World health survey in Brazil, 2003. Cad Saude Publica 2005; 21 Suppl: 4-5.

17. Brasil. Ministério da Saúde. Centro Nacional de Epidemiologia (CENEPI). Proposta para os Inquéritos Nacionais em Saúde, 2000. Mimeo.

18. Malta DC, Cesáreo AC, Moura L; Morais Neto OL; Silva Júnior JB. A construção da vigilância e prevenção das doenças crônicas não transmissíveis no contexto do Sistema Único de Saúde. Epidemiol Serviços Saúde 2006; 15: 47-65.

19. Brasil. Ministério da Saúde. Secretaria de Vigilância em Saúde; Instituto Nacional do Câncer. Inquérito domiciliar sobre comportamentos de risco e morbidade referida de doenças e agravos não transmissíveis: Brasil, dezessete capitais e Distrito Federal, 2002-2005. 2006. Acessado em dezembro de 2007 em http:/www.inca.gov.br.

20. Brasil. Ministério da Saúde. Vigitel Brasil 2006: vigilância de fatores de risco e proteção para doenças crônicas por inquérito telefônico. Brasilia, 2007.297 p.

21. Malta DC, Castro, A; Silva, MMA; Mascarenhas, MDM; Neto Morais Neto OL. O desafio contemporâneo da violência e a promoção da saúde. Revista Médica de Minas Gerais 2006; 16 (4, supl 2): 72-79.

22. Brasil. Ministério da Saúde. Portaria $n^{\circ} .3175,18$ de dezembro de 2006. Institui Comitê Gestor com a finalidade de acompanhar e coordenar as demandas de pesquisas relativas a inquéritos populacionais, que sejam definidos como de interesse. Diário Oficial da União, Poder Executivo. Brasília, DF, 19 de dezembro, 2006. Seção I, p. 145.

23. Quintslr, MMM. Sistema Integrado de pesquisas Domiciliares do IBGE. Comunicação Coordenada na I Reunião do Grupo de inquéritos populacionais. Ministério da Saúde. Secretaria de Vigilância em Saúde, 2007. Mimeo.

24. Kennedy J; Morgan S - Health care access in three nations: Canada, insured America, and uninsured America. Int J Health Ser 2006; 36 (4): 697-717. 